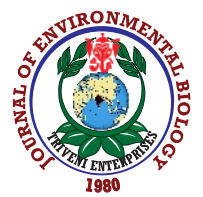

\title{
An investigation of environmental factors influencing quantitative accuracy and recognition rate of craniofacial reconstructions
}

\author{
S. M. Kim1', W. J. Lee', J. H. Cho ${ }^{3}$, C. M. Wilkinson', C. U. Choi ${ }^{2}$ and S. S. Lee ${ }^{1 *}$ \\ 'Medical Examiner's Office, National Forensic Service, Wonju-26460, Republic of Korea \\ ${ }^{2}$ Department of Forensic Medicine Investigation, National Forensic Service Seoul Institute, Seoul-08036, Republic of Korea \\ ${ }^{3}$ Department of Orthodontics, School of Dentistry, Chonnam National University, Gwangju-61186, Republic of Korea \\ ${ }^{4}$ Face Lab, Liverpool John Moores University, IC1 Liverpool Science Park, Liverpool-L35TF, United Kingdom \\ *Corresponding Author Email: sslee1418@gmail.com
}

\section{Abstract}

Aim: The accuracy of forensic craniofacial reconstruction/approximation can be influenced by a number of environmental factors. The aim of this study was to investigate how different average facial soft tissue depth datasets might influence the accuracy of craniofacial reconstruction employing two accuracy assessment methods;face-pool comparison and geometric surface comparison.

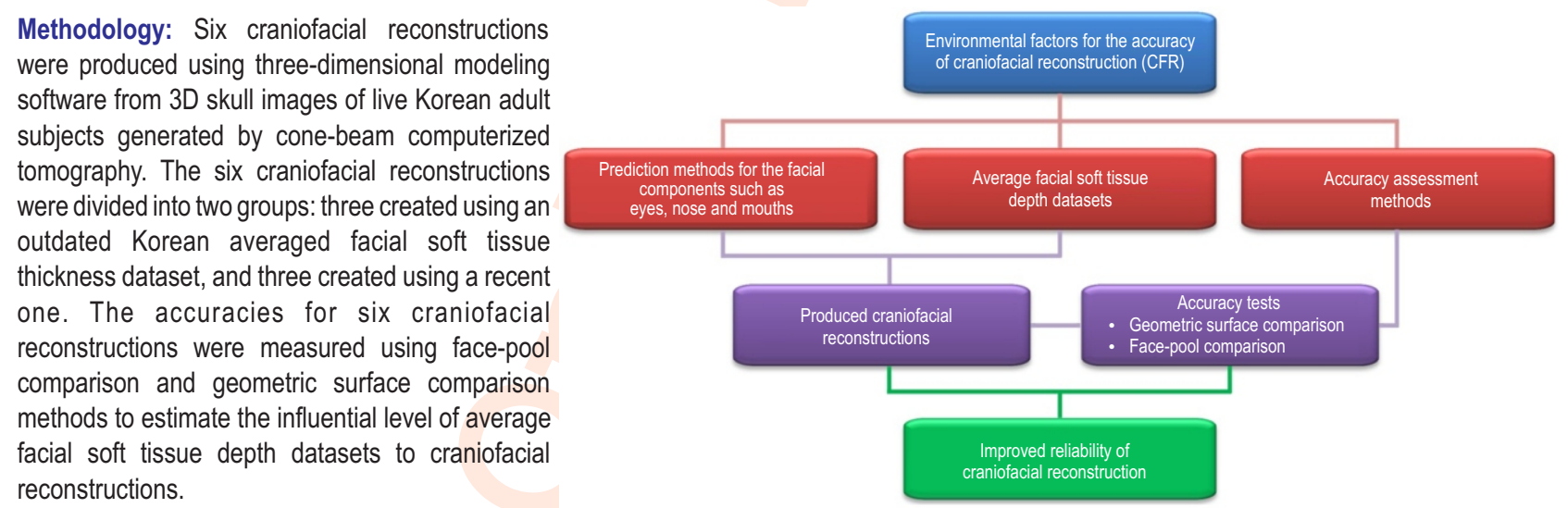

Results: In face-pool comparisons, the recent dataset demonstrated a higher recognition rate than the outdated one. The results were compared to those of geometric surface comparisons using the same six craniofacial reconstructions; a positive correlation was detected.

Interpretation: The results suggest that appropriate averaged facial soft tissue depth datasets influence the accuracy of craniofacial reconstructions, and that the geometric surface comparison method might be interchangeable with face-pool comparison method to evaluate the accuracy of craniofacial reconstructions.

Key words: Accuracy, Craniofacial reconstruction, Environmental factors, Geometric surface, Recognition rate

How to cite : Kim, S.M., W.J. Lee, J.H. Cho, C.M. Wilkinson, C.U. Choi and S.S. Lee: An investigation of environmental factors influencing quantitative accuracy and recognition rate of craniofacial reconstructions. J. Environ. Biol., 41, 539-548 (2020). 


\section{Introduction}

Craniofacial reconstruction (CFR), also known as "facial approximation" (Stephan, 2003; Wilkinson, 2005), enables projection of the facial appearance of a person onto a skull based on both scientific standards and artistic skill (Wilkinson, 2010). Nevertheless, the ultimate aim of CFR is to predict antemortem facial appearance to facilitate identification of an individual in the forensic sciences. The resemblance of the reconstructed face to the antemortem facial countenance determines the reliability of technique. The accuracy of CFR is mainly influenced by predicting of facial components based on morphological/ craniometrical skull analyses and averaged facial soft tissue thickness (FSTT) data (Lee et al., 2012). The prediction guidelines for the eyes (Wilkinson and Mautner, 2003; Kim et al., 2016), nose (Lee et al., 2014; TedeschiOliveira et al., 2016; Utsuno et al., 2016), and mouth (Wilkinson et al., 2003; Dias et al., 2016; Mala and Veleminska, 2016) have been updated. In addition, various averaged FSTT datasets have been established for diverse ethnic groups (Hamid and Abuaffan, 2016; Wang et al., 2016).

To guarantee reliability, the accuracy of CFR in terms of degree of resemblance between the actual and reconstructed face must be tested by established qualitative and quantitative assessments (Lee et al., 2012). The former is done mainly using one-to-one and face-pool comparison methods (Lee et al., 2012; Stephan and Arthur, 2006; Stephan and Cicolini, 2008). In the one-to-one comparison method, assessors are instructed to determine the degree of resemblance between CFR and corresponding photograph of the face. In the facepool comparison method, assessors are instructed to match a target image of CFR to a range of faces in a photograph array, which is similar to an identification parade. Quantitative approaches include anthropometric comparison of discrepancies in primary landmarks between CFR and target face (Short et al., 2014), and superimposition of CFR on a photograph of the target face to identify differences in the surface contours of two head models (Wilkinson, 2010).

Face-pool comparison is considered an efficient and objective approach (Stephan and Arthur, 2006; Stephan and Cicolini, 2008; Lee and Wilkinson, 2016). Although classified as a qualitative approach because the accuracy of CFR is determined by the subjective decisions of the assessors, it is realistic because CFRs in actual forensic cases are recognized by acquaintances based on facial perception (Stephan and Cicolini, 2008; Lee and Wilkinson, 2016; Bruce et al., 1999). Furthermore, the results can be presented as numerical recognition rates. Therefore, this method is used in CFR accuracy studies to assess the objective accuracy of reconstructed faces in association with facial recognition (Stephan and Arthur, 2006; Stephan and Cicolini, 2008; Lee and Wilkinson, 2016). The first case was reported by Snow et al. (1970), who produced male and female CFRs from the skulls of a body donor and a forensic case, and explored the accuracy of reconstruction by face-pool comparison.
Subsequently, numerous studies of accuracy of CFR have used face-pool comparison (Stephan and Arthur, 2006; Stephan and Cicolini, 2008; Lee and Wilkinson, 2016; Snow et al., 1970; Stephan and Cicolini 2010). Although face-pool comparison enables evaluation of the validity of CFR, it is a complicated and time-consuming procedure involving acquisition and selection of facial photographs for distractors, arrangement of the photographs in an array, recruitment of assessors, and a questionnaire survey (Lee and Wilkinson, 2016).

Thus, alternative objective methods of evaluating the accuracy of CFR are required. Development of computerized facial depiction systems has enabled computer-generated threedimensional (3D) CFR (Stephan, 2003; Wilkinson et al., 2006; Guyomarc'h et al., 2014). Using such systems, a CFR can be produced using an automated (Guyomarc'h et al., 2014; Turner et al., 2005; Claes et al., 2010; Decker et al., 2013) or modeling (virtual sculpture) method (Short et al., 2014; Lee and Wilkinson, 2016; Wilkinson et al., 2006; Wilkinson, 2003). The accuracy of computer-generated CFRs can be measured using 3D geometric surface analyses (reverse modeling software) as the 3D CFRs can be aligned in a $3 D$ virtual space by reverse modeling software. Differences in skin thickness can be quantified and visualized as a deviation map (Lee et al., 2012; Wilkinson et al., 2006; Guyomarc'h et al., 2014; Lee et al., 2015).

Wilkinson et al. (2006) used the geometric surface comparison method to evaluate the accuracy of two CFRs produced from computed tomography (CT)-scanned skull models of two live individuals (white North American male and female). Lee et al. (2012; 2015) performed two CFR accuracy studies using the geometric surface comparison method. First study (Lee et al., 2012) employed the 3D-scanned skull models of three live Korean subjects for CFRs (hereafter, 'outdated FSTT'), and used the same methodology as an earlier study (Wilkinson, 2006). The average FSTT data for CFRs (Lee et al., 2012) were obtained from a Korean population in Russia whose ancestors immigrated 100 years ago (Lebedinskaya et al., 1993).

Second study (Lee et al., 2015) evaluated the accuracy of CFRs using an updated FSTT dataset of the current Korean population (Hwang et al., 2012) (hereafter, 'recent FSTT'). The results were compared to investigate the influence of FSTT data based on the accuracy of CFR (Lee et al., 2012; Lee et al., 2015). However, the three aforementioned studies had important limitation (Lee et al., 2012; Wilkinson et al., 2006; Lee et al., 2015). Whether improved quantitative accuracy of CFR increases the recognition rate is unclear. Therefore, the effects of improved accuracy of CFR on the target face recognition rate should be investigated using some other quantitative method. In this study, the influence of FSTT dataset was evaluated based on the CFR and the recognition rate in each CFR tested by the facepool comparison method. In addition, the results were compared to those of previous CFR accuracy studies using the geometric surface comparison method to assess whether improved CFR accuracy is correlated to increased recognition rate. 


\section{Materials and Methods}

Six CFRs (two sets of three CFRs produced from outdated FSTT(Lee et al., 2012) and recent FSTT (Lee et al., 2015) were subjected to face-pool comparison. These 3D skull images from six living Korean adult subjects were acquired by cone-beam computed tomography (CBCT) (Alphard Vega ${ }^{\mathrm{TM}}$, Asahi Roentgen Co., Kyoto, Japan) with a voxel size of $0.39 \mathrm{~mm}$ and field of view (FOV) of $200 \times 179 \mathrm{~mm}$ (Table 1). Six CFRs using the corresponding 3D skull models were produced using 3D modeling software (Geomagic $\circledast$ FreeForm $\circledast P l u s, 3 D$ Systems $®$, USA). Two FSTT datasets for adult Koreans were applied to generate the CFRs; an outdated dataset (Lebedinskaya et al., 1993) and a recent dataset (Hwang et al., 2012). The faces of subjects were rebuilt according to combination method (Stephan etal., 2003; Lee etal., 2012; Lee etal., 2015).

To assess accuracy using face-pool comparison method, six CFRs were divided into two groups: three CFRs using the outdated Korean average FSTT dataset (Lebedinskaya et al., 1993) (CFR-A, CFR-B and CFR-C) (Fig. 1), and three using the recently generated Korean average FSTT dataset (Hwang et al., 2012) (CFR-D, CFR-E and CFR-F) (Fig. 2). The recognition rates of the six CFRs were evaluated using the face-pool comparison method. To evaluate the recognition rate, six photograph arrays were prepared, each including 10 facial photographs: 9 distractors and 1 matching the CFR.

Distractors were selected according to the method for constructing line-ups (distractors) recommended by the Police and Criminal Evidence Act 1984 code D of the UK government (Home Office, 2017). In the first step, 111 frontal facial photographs of volunteers ( 55 of males and 56 of females) were taken. The volunteers were informed of the purpose of the study and provided consent for use of their facial photographs in face pools and publications. These facial photographs were candidate distractors. Distractors for each CFR were selected by five assessors by rating the degree of resemblance from one (lowest resemblance) to five (highest resemblance) between the actual facial photograph and the candidate distractors of the same sex. Hence, each CFR had 55 (male) or 56 (female) distractor candidates. The final degree of resemblance was taken as that most frequently recorded by the five assessors. This process resulted in six sets of candidate distractors arranged by the degree of resemblance.
In the second step, the resemblance-rated candidate distractors were divided into five groups according to their degree of resemblance. Two distractors were randomly selected from each group, with the exception of the group with a resemblance rating of five, from which only one distractor was selected. As a result, nine distractors were selected for each photograph array. Because CFRs do not include hair, the hairstyles were removed from the facial photographs as much as possible. The photographs were black and white. To ensure that the quality of facial image did not affect the evaluation, the image brightness, contrast, background, and size were homogenized using imageediting software (Adobe ${ }^{\circledR}$ Photoshop $®$ CS6, ver. 13.0). The 10 facial photographs were arranged randomly in a photograph array (Fig. 3). Face-pool recognition performance was evaluated using an online survey tool (http://www.surveymonkey.com ${ }^{\mathrm{TM}}$ ). The questionnaires were distributed to acquaintances of the researchers by e-mail or messaging software. By clicking on a hyperlink, participants (assessors) started the survey after reading an information sheet and providing consent to participate. Participants received six questionnaires, each of which consisted of one target CFR and 10 facial photographs, and were instructed to select the face they felt corresponded to the CFR. Participants could select 'No actual face' if they decided that there was no matching face. There was no time limit and all answers were anonymous. Participants did not provide any information other than their sex, age, nationality, and occupation related to craniofacial analyses. In all, 164 answers were collected. The results of the questionnaire were divided according to the outdated Korean average FSTT dataset and the recently generated Korean average FSTT dataset.

Statistical analyses: Statistical analyses were performed using $R$ ver. 3.3.1 software. Pearson's Chi-square and goodness-of-fit tests, paired t-tests, one-way analyses of variance (ANOVA), and Kruskal Wallis tests were performed to assess the correlations between variables. The level of significance (a) was set at 0.05 . This study was approved by the Institutional Review Board of Chonnam National University Dental Hospital (Approval number: CNUDH-2017-010).

\section{Results and Discussion}

The sex, age, and occupation of the assessors are shown in Table 2. In all, 164 responses were collected from 72 (43.9\%) male and $92(56.1 \%)$ female assessors. The age distribution was

Table 1: Profiles of six subjects on which three-dimensional computed tomography scanned skull models were based

\begin{tabular}{lllll}
\hline Study & CFR & Sex & Age & Applied average FSTT dataset \\
\hline Lee et al. (2012) (outdated FSTT) & CFR-A & Male & 30 & Lebedinskaya et al. (1993) \\
& CFR-B & Female & 27 & \\
Lee et al. (2015) (recent FSTT) & CFR-C & Male & 27 & Hwang et al. (2012) \\
& CFR-D & Male & 27 & \\
& CFR-E & Female & 32 & \\
\hline
\end{tabular}






Fig. 1: Three CFRs used for outdated FSTT (left to right, CFR-A, CFR-B, and CFR-C).

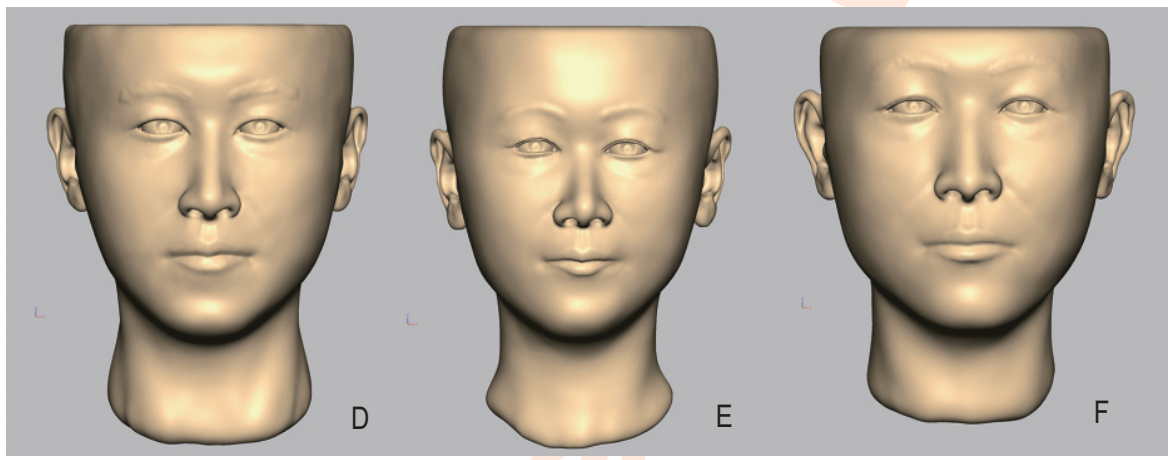

Fig. 2: Three CFRs used for recent FSTT (left to right, CFR-D, CFR-E, and CFR-F).

as follows: $72(43.9 \%)$ were in their 20 s, $54(32.9 \%)$ were in their 30 s, $29(17.7 \%)$ were in their 40 s, $8(4.9 \%)$ were in their 50 s, and 1 $(0.6 \%)$ was more than 60 years of age. The occupations of 29 $(17.7 \%)$ assessors were related to craniofacial analyses.

The assessors had $50 \%$ chance of correctly determining whether the target was present in the face pool, and $10 \%$ chance of choosing the correct face if they decided that the target was present in the face pool. Therefore, each target had $5 \%$ chance $(50 \% \times 10 \%)$, as all face-pool comparisons were target-present trials. The recognition rates for six CFRs were greater than would be expected by chance $(23.2 \%$ for CFR-A, $34.2 \%$ for CFR-B, 9.2\% for CFR-C, $26.2 \%$ for CFR-D, $45.1 \%$ for CFR-E, and $20.7 \%$ for CFR-F; Table 3, Fig. 4). The overall mean recognition rate was $26.4 \%$. All results were significant $(p<0.001)$, which indicates that the decisions of the assessors were not due to chance (Table 3). CFR-A, CFR-B, and CFR-E had the highest recognition rates among the 10 facial photographs in each photograph array, followed by CFR-D, CFR-F and CFR-C. The overall mean recognition rates of male and female assessors were $25.7 \%$ and $27.0 \%$, respectively; the difference was not significant. Assessors in their 20s, 30s, 40s, 50s and 60s showed recognition rates of $29.4 \%, 20.7 \%, 28.2 \%, 29.2 \%$ and $50.0 \%$, respectively. The result from 60 s was scored by only one assessor. Assessors with occupations related and not related to craniofacial analyses had a recognition rate of $22.4 \%$ and $27.3 \%$, respectively $(p>0.05)$. Next, the results were analyzed to note whether the CFR was produced using the dataset from outdated FSTT (Lee et al., 2012) or recently FSTT (Lee et al., 2015). The mean recognition rates were $22.2 \%$ and $30.7 \%$ for outdated FSTT and recent FSTT, respectively (Table 4).

Table 2: Sex, age and occupation of the assessors

\begin{tabular}{ll}
\hline Categories of assessor & Proportion (\%) \\
\hline Sex & $72(43.9 \%)$ \\
Male & $92(56.1 \%)$ \\
Female & \\
Age (years) & $72(43.9 \%)$ \\
20 s & $54(32.9 \%)$ \\
30 s & $29(17.7 \%)$ \\
40 s & $8(4.9 \%)$ \\
$50 s$ & $1(0.6 \%)$ \\
More than 60 & \\
Occupation & $29(17.7 \%)$ \\
Relevant to craniofacial analyses & $135(82.3 \%)$ \\
Irrelevant to craniofacial analyses &
\end{tabular}




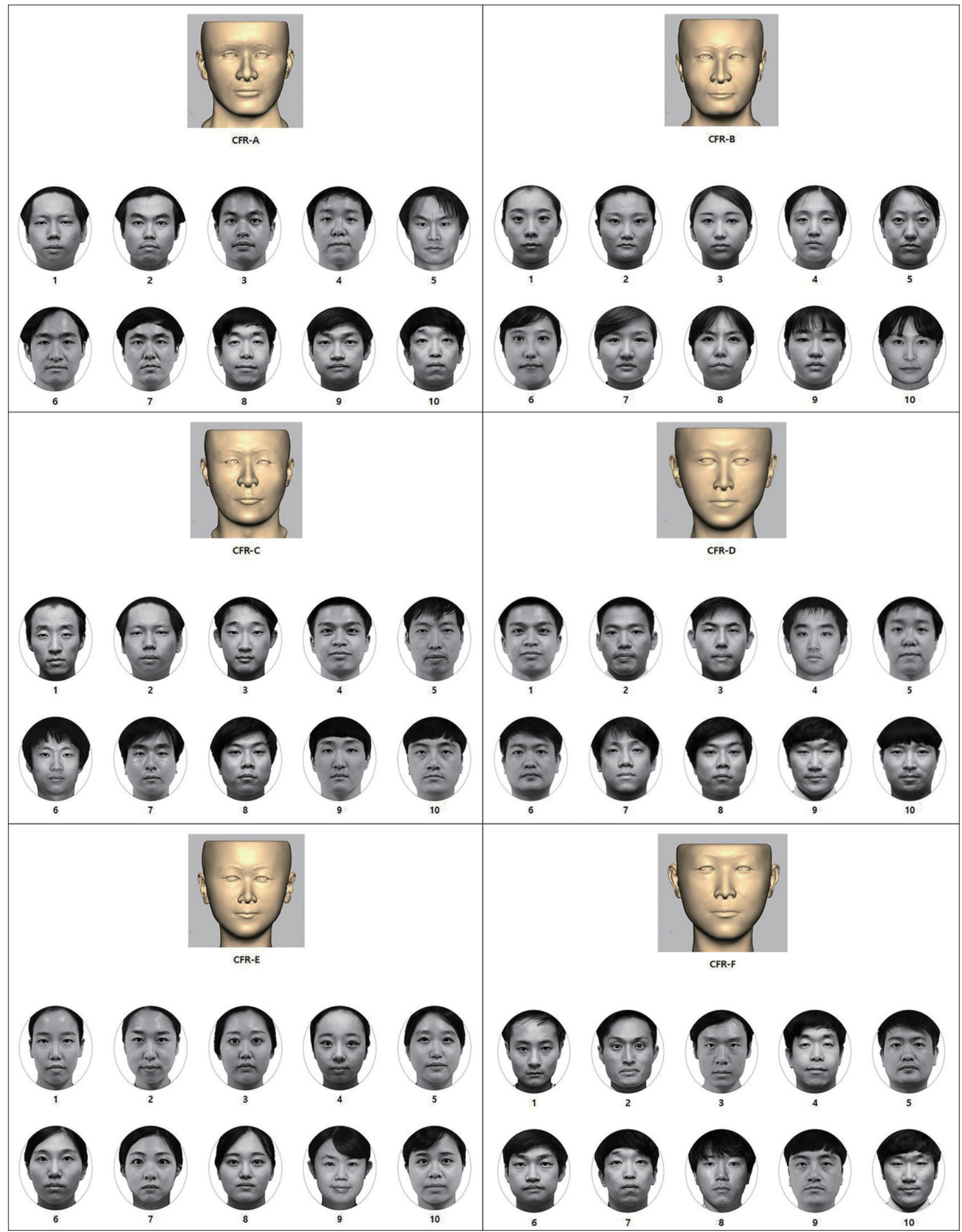

Fig. 3: Photograph arrays used to assess CFR recognition rates. 
Table 3: Overall recognition rates for six CFRs

\begin{tabular}{llll}
\hline CFR & Hit count/total number of responses & Recognition rate $(\%)$ & Chi-square goodness-of-fit test \\
\hline CFR-A & $38 / 164$ & 23.2 & $p<0.001$ \\
CFR-B & $56 / 164$ & 34.2 & $p<0.001$ \\
CFR-C & $15 / 164$ & 9.2 & $p<0.001$ \\
CFR-D & $43 / 164$ & 26.2 & $\mathrm{p}<0.001$ \\
CFR-E & $74 / 164$ & 45.1 & $\mathrm{p}<0.001$ \\
CFR-F & $34 / 164$ & 20.7 & $\mathrm{p}<0.001$ \\
Mean & & 26.4 & \\
\hline
\end{tabular}

Table 4: Recognition rates for outdated FSTT and recent FSTT

\begin{tabular}{lllll}
\hline Group & CFR & $\begin{array}{l}\text { Recognition } \\
\text { rate }(\%)\end{array}$ & $\begin{array}{l}\text { Mean recognition } \\
\text { rate }(\%)\end{array}$ & $\begin{array}{l}\text { Significance } \\
(\boldsymbol{p} \text {-value })\end{array}$ \\
\hline Lee et al. (2012) & CFR-A & 23.2 & & \\
(outdated FSTT) & CFR-B & 34.2 & & \\
& CFR-C & 9.2 & & $0.0015^{*}$ \\
Lee et al. (2015) & CFR-D & 26.2 & 30.7 & \\
(recent FSTT) & CFR-E & 45.1 & & \\
& CFR-F & 20.7 & & \\
\hline
\end{tabular}

${ }^{*} p<0.05$

Table 5: Recognition rates of male craniofacial reconstructions (CFRs)

\begin{tabular}{lllll}
\hline Group & CFR & $\begin{array}{l}\text { Recognition } \\
\text { rate }(\%)\end{array}$ & $\begin{array}{l}\text { Mean recognition } \\
\text { rate }(\%)\end{array}$ & $\begin{array}{l}\text { Significance } \\
(p \text {-value })\end{array}$ \\
\hline Lee et al. (2012) & CFR-A & 23.2 & 16.2 & $0.012^{*}$ \\
(outdated FSTT) & CFR-C & 9.2 & 23.5 & \\
Lee et al. (2015) & CFR-D & 26.2 & 20.7 & \\
(recent FSTT) & CFR-F & 20.7 & \\
\hline
\end{tabular}

${ }^{*} p<0.05$

The difference between the two groups was significant $(p$ $<0.05$ ), except for 60 s whose recognition rate was collected by only one assessor. The two female CFRs showed recognition rates of $34.2 \%$ for CFR-B for outdated FSTT and $45.1 \%$ for CFR$\mathrm{E}$ for recent $\mathrm{FSTT}$. A higher recognition rates of female CFRs may have influenced the results, subanalyses according to sex were conducted. The four male CFRs were divided according to whether the CFR was produced from outdated FSTT or recent FSTT, and analyzed. The two male CFRs for recent FSTT had a higher recognition rate $(23.5 \%)$ than the two male CFRs foroutdated FSTT $(16.2 \%)(p<0.05)$ (Table 5).Similarly, the female CFRs for recent FSTT had a higher recognition rate $(45.1 \%)$ than the female CFR for outdated FSTT $(34.3 \%)(p<$ 0.05) (Table 6). The face-pool comparison results were compared to those of two previous quantitative accuracy studies (Lee et al., 2012; Lee et al., 2015) to determine whether improved quantitative accuracy is correlated to increased CFR recognition rate. In geometric surface comparisons, $65.2 \%$ and $84.5 \%$ of the CFR surface deviated from the actual facial scan by no more than $\pm 2.5 \mathrm{~mm}$ for outdated FSTT and recent FSTT, respectively (Table 7). The increased quantitative accuracy from outdated FSTT to recent FSTT (65.2\% to $84.5 \%$ ) was positively correlated with the face-pool comparison results (22.2\% to $30.7 \%$ ) (Figs. 5 and 6$)$. The results using recent FSTT showed a better quantitative accuracy and lesser deviation than those using outdated FSTT (Fig 5). The face-pool comparison results exhibited a similar pattern (Fig. 6), although the discrepancy between the two studies (8.5\%) was of lesser magnitude than the results from the quantitative studies (19.3\%).

The overall mean recognition rate for six CFRs of $26.4 \%$ was lower. However, it is comparable to those obtained in previous studies that used recognition tests: $12.7 \%$ (Lee and Wilkinson, 2016), 23\% (Fernandes et al., 2012), 21\% (Stephan and Arthur, 2006) and $21 \%$ (Stephan and Cicolini, 2008). Although the studies employed slightly different methodologies and performance procedures, the mean recognition rate in this study was higher than previous studies. The mean recognition rate recorded by familiar- 


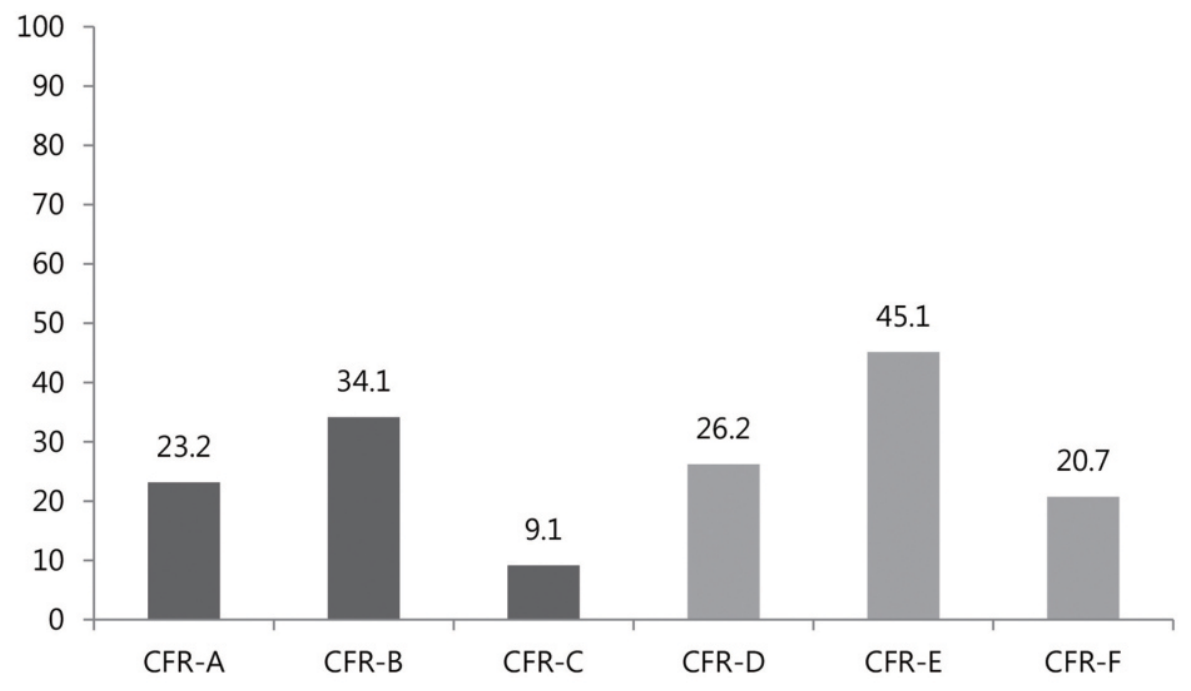

Fig. 4: Overall recognition rates of six CFRs.



Fig. 5: Box plot of difference between outdated FSTT and recent FSTT by geometric surface comparison.

race assessors for CFRs was 19.4\% (Lee and Wilkinson, 2016), who employed almost the same methodology as in the current study in terms of CFR ancestry and assessors. It might be assumed that the CFR method has enhanced accuracy when comparing the result of Lee and Wilkinson (2016). However, the efforts to increase the accuracy of CFR must be continued as general recognition rates have remained about one-quarter of the recognition rate.

The mean recognition rates did not significantly differ between male and female assessors, in agreement with previous reports (Lee and Wilkinson, 2016; Snow et al., 1970; Wilkinson et 
Table 6: Recognition rates of the female craniofacial reconstructions (CFRs)

\begin{tabular}{llll}
\hline Group & CFR & Recognition rate (\%) & Significance $(p$-value $)$ \\
\hline Lee et al. (2012) (outdated FSTT) & CFR-B & 34.2 & \\
Lee et al. (2015) (recent FSTT) & CFR-E & 45.1 & $0.028^{*}$ \\
\hline
\end{tabular}

${ }^{*} p<0.05$

Table 7: Results of the geometric surface and face-pool comparative methods

\begin{tabular}{llll}
\hline CFR & & $\begin{array}{l}\text { Geometric surface } \\
\text { comparison (\%) }\end{array}$ & $\begin{array}{l}\text { Recognition rate in face- } \\
\text { pool comparison (\%) }\end{array}$ \\
\hline Lee et al. (2012) (outdated FSTT) & CFR-A & 54.3 & 23.2 \\
& CFR-B & 64.6 & 34.2 \\
& CFR-C & 76.7 & 9.2 \\
Lee et al. (2015) (recent FSTT) & Mean & 65.2 & 22.2 \\
& CFR-D & 87.5 & 26.2 \\
& CFR-E & 79.3 & 45.1 \\
& CFR-F & 86.8 & 20.7 \\
\hline
\end{tabular}

${ }^{*}$ Geometric surface comparison: Distribution (\%) of the deviation error (minimum range within $2.5 \mathrm{~mm}$ ) between the surfaces of the CFR and the face of the corresponding subject

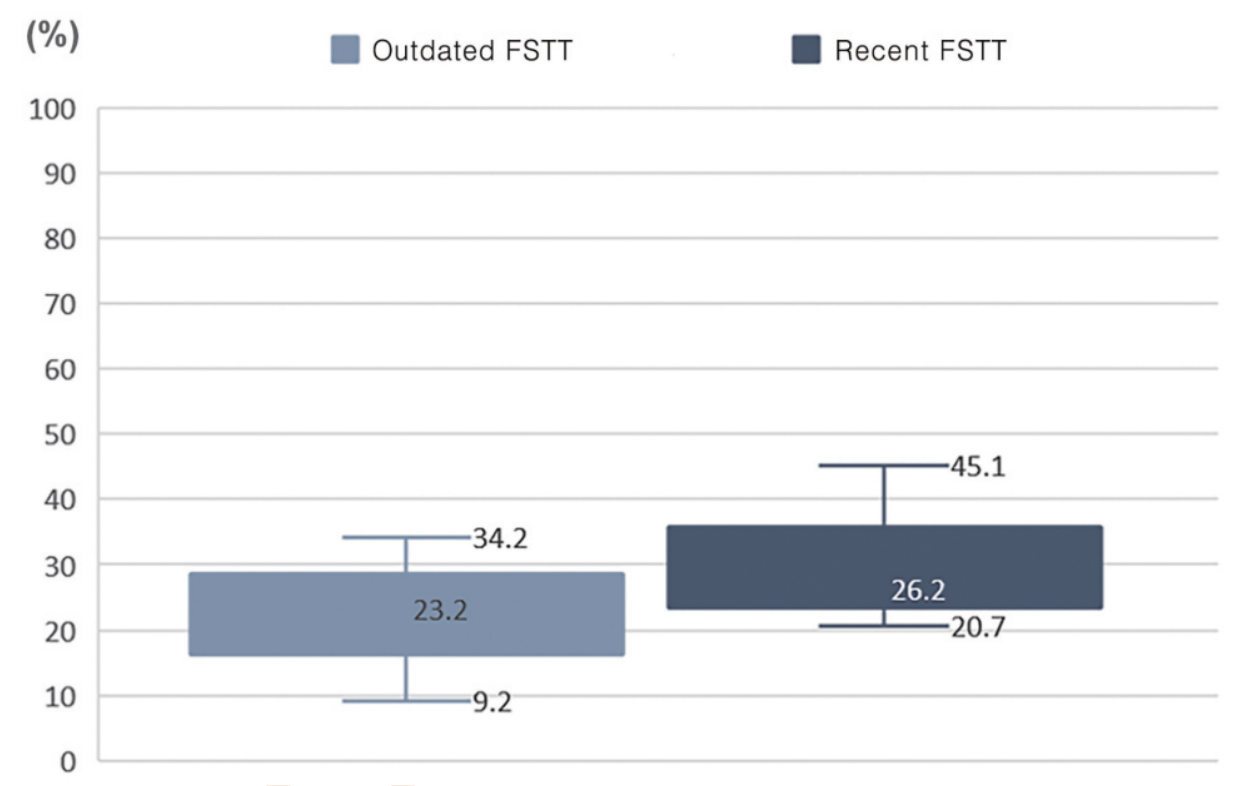

Fig. 6: Box plot of the difference between outdated FSTT and recent FSTT by face-pool comparison.

al., 2006). Also, assessor age did not affect the CFR recognition rate with the exception of one assessor who was more than 60 years of age. The recognition rate did not significantly differ between assessors with occupations related and not related to craniofacial analyses. This contradicts two previous studies (Snow et al., 1970; Wilkinson and Whittaker, 2002) that utilized face-pool comparison. Snow et al. (1970) reported that police officers experienced in facial identification had a significantly higher CFR recognition rate (80.2\%) than general public (56.9\%). Wilkinson and Whittaker (2002) reported that experts in facial analyses had a significantly higher CFR recognition rate $(60 \%)$ than non-experts (36\%). A possible hypothesis could be that craniofacial identification was introduced relatively recently in South Korea. It may be assumed that the assessors related to 
craniofacial analyses have less training and/or experience in craniofacial identification than their colleagues in other nations. However, this hypothesis must be carefully interpreted until continuous recognition survey is performed.

The correlation between use of appropriate average FSTT data and the quantitative accuracy/recognition level of CFR has been investigated by researchers. Wilkinson et al. (2002a) produced six CFRs using six FSTT datasets from six groups with different ancestries, and used a resemblance rating method and quantitative visual analyses to assess the recognition rate. Use of an appropriate FSTT dataset yielded the highest accuracy. Fernandes et al. (2012) using the face pool comparison method evaluated the recognition rate of three Brazilian CFRs using three average FSTT datasets from a Brazilian MRI study, Brazilian cadaver study, and white Americans. The CFR using FSTT dataset from the Brazilian MRI study scored the highest recognition rate. Both studies demonstrated that a suitable FSTT dataset influences the recognition rate. Our results are in agreement with those results.

In terms of correlation between quantitative accuracy and recognition rate, Wilkinson et al. (2006), using the geometric surface comparison method, demonstrated that $60 \%$ and $52 \%$ of the surface of white European male and female CFRs deviated from the actual values by no more than $\pm 2.5 \mathrm{~mm}$. The face-pool comparison method had a recognition rate of $69 \%$ for the male CFR and $71 \%$ for the female CFR. Lee et al. (2012; 2015) evaluated CFR accuracy using the geometric surface comparison method and an outdated FSTT dataset (Lebedinskaya et al., 1993) and reported equivalent or improved deviations between the CFRs and the targets $(54 \%, 65 \%$, and $77 \%$ of the surfaces of three CFRs deviated from the facial scans by no more than $\pm 2.5 \mathrm{~mm}$ ) than the results of Wilkinson et al. (2006). In Lee et al. (2015), which used recent FSTT dataset for Korean adults (Hwang et al., 2012), the deviations between the CFRs and the target subjects were significantly greater $(88 \%, 79 \%$ and $87 \%$ of the surfaces of the three CFRs deviated from the facial scans by no more than $\pm 2.5 \mathrm{~mm}$ ) than those for outdated FSTT. However, whether improved quantitative accuracy increases the CFR recognition rate was unclear in the previous studies (Lee et al., 2012; Lee et al., 2015; Fernandes et al., 2012; Wilkinson et al., 2002b).

In this study, we evaluated the accuracy of CFR using the face-pool comparison method; the mean recognition rates were $22.15 \%$ and $30.69 \%$ for CFRs in outdated FSTT and recent FSTT, respectively. This represents a 1.4-fold increase for recent FSTT. The mean rates of a no more than $\pm 2.5 \mathrm{~mm}$ deviation between the surfaces of the CFRs and the corresponding subjects were $65.2 \%$ and $84.5 \%$ in outdated FSTT and recent FSTT, respectively. This represents a 1.3-fold increase for recent FSTT. The results of the geometric-surface and face pool comparative methods demonstrated a positive correlation. However, it was limited to the overall mean values. Another limitation of this study was that different skulls were used in the outdated FSTT and recent FSTT studies, so the individuality of the skulls could have influenced the results. Therefore, a future study should establish a correlation between quantitative accuracy and recognition rate employing CFRs produced from the same skull with a different average FSTT dataset.

The face-pool comparison method enabled assessment of the influence of FSTT dataset used to generate the CFRs on the recognition rate. Although this study has limitations, the results suggest that quantitative accuracy could be converted into the recognition rate. Therefore, our findings suggest that the geometric-surface comparison method could be used to evaluate the CFR recognition rate. The face-pool comparison method is complex and time-consuming, and so geometric surface comparison will be an alternative method to evaluate the CFR recognition rate quantitatively and qualitatively.

In conclusion, the CFRs generated using a recent Korean FSTT dataset had higher recognition rates than did CFRs generated using an outdated dataset; this is in agreement with previous reports. Overall, the results indicate that improved quantitative accuracy translates into a higher CFR recognition rate. In addition, the geometric surface comparison method could be an alternative to the face-pool comparison method for assessing both quantitative and qualitative accuracy of CFR. This tentative conclusion should be verified clearly in further studies.

\section{Acknowledgments}

This research was supported by Basic Science Research Program through the National Research Foundation of Korea (NRF) funded by the Ministry of Science and ICT (NRF2018R1A1A1A05021116).

\section{References}

Bruce, V., Z. Henderson, K. Greenwood, P.J.B. Hancock, A.M. Burton and P. Miller: Verification of face identities from images captured on video. J. Exp. Psychol. Appl., 5, 339-360 (1999).

Claes, P., D. Vandermeulen, S. De Greef, G. Willems, J.G. Clement and P. Suetens: Computerized craniofacial reconstruction: Conceptual framework and review. Forensic Sci. Int., 201, 138-145 (2010).

Decker, S., J. Ford, S. Davy-Jow, P. Faraut, W. Neville and D. Hilbelink: Who is this person? A comparison study of current threedimensional facial approximation methods. Forensic Sci. Int., 229, 161.e1-e8 (2013).

Dias, P.E., G.E. Miranda, T.L. Beaini and R.F. Melani: Practical application of anatomy of the oral cavity in forensic facial reconstruction. PLoS One, 11, e0162732 (2016).

Fernandes, C.M.S., M. da Costa Serra, J.V.L. da Silva, P.Y. Noritomi, F.D.A. de Sena Pereira and R.F.H. Melani: Tests of one Brazilian facial reconstruction method using three soft tissue depth sets and familiar assessors. Forensic Sci. Int., 214, 211.e1-e7 (2012).

Guyomarc'h, P., B. Dutailly, J. Charton, F. Santos, P. Desbarats and H. Coqueugniot: Anthropological facial approximation in three dimensions (AFA3D): Computer-assisted estimation of the facial morphology using geometric morphometrics. J. Forensic Sci., 59, 1502-1516 (2014). 
Hamid, S. and A.H. Abuaffan: Facial soft tissue thickness in a sample of Sudanese adults with different occlusions. Forensic Sci. Int., 266, 209-214 (2016).

Home Office: Police and criminal evidence act 1984: Code D Revised Code of Practice for the identification of persons by Police Officers. https://www.gov.uk/government/uploads/system/uploads/attach ment_data/file/592562/pace-code-d-2017.pdf, 2017 (accessed 12 January 2018).

Hwang, H.S., M.K. Park, W.J. Lee, J.H. Cho, B.K. Kim and C.M. Wilkinson: Facial soft tissue thickness database for craniofacial reconstruction in Korean adults. Forensic Sci. Int., 57, 1442-1447 (2012).

Kim, S.R., K.M. Lee, J.H. Cho and H.S. Hwang: Three-dimensional prediction of the human eyeball and canthi for craniofacial reconstruction using cone-beam computed tomography. Forensic Sci. Int., 261, 164.e1-e8 (2016).

Lebedinskaya, G.V., T.S. Balueva and V.S. Veselovaskaya: Principles of facial reconstruction. In: Forensic Analysis of the Skull: Craniofacial Analysis, Reconstruction and Identification, (Eds.: M.Y. Işcan and R.P. Helmer). Wliey Liss Publishers, New York, pp. 183-198(1993).

Lee, K.M., W.J. Lee, J.H. Cho and H.S. Hwang: Three-dimensional prediction of the nose for facial reconstruction using cone-beam computed tomography. Forensic Sci. Int., 236, 194.e1-e5 (2014).

Lee, W.J. and C.M. Wilkinson: The unfamiliar face effect on forensic craniofacial reconstruction and recognition. Forensic Sci. Int., 269, 21-30 (2016).

Lee, W.J., C.M. Wilkinson and H.S. Hwang: An accuracy assessment of forensic computerized facial reconstruction employing ConeBeam Computed Tomography from live subjects. J. Forensic Sci., 57, 318-27 (2012).

Lee W.J., C.M. Wilkinson, H.S. Hwang and S.M. Lee: Correlation between average tissue depth data and quantitative accuracy of forensic craniofacial reconstructions measured by geometric surface comparison method. J. Forensic Sci., 60, 572-80 (2015).

Mala, P.Z. and J. Veleminska: Vertical lip position and thickness in facial reconstruction: A validation of commonly used methods for predicting the position and size of lips. J. Forensic Sci., 61, 10461054 (2016)

Short, L.J., B. Khambay, A. Ayoub, C. Erolin, C. Rynn and C.M. Wilkinson: Validation of a computer modelled forensic facial reconstruction technique using CT data from live subjects: A pilot study. Forensic Sci. Int., 237, 147.e1-e8 (2014).

Snow, C.C., B.P. Gatliff and K.R. McWilliams: Reconstruction of facial features from the skull: An evaluation of its usefulness in forensic anthropology. Am. J. Phys. Anthropol., 33, 221-228 (1970).

Stephan, C.N.: Anthropological facial 'reconstruction' - recognizing the fallacies, 'unembracing' the errors, and realizing method limits. Sci. Justice., 43, 193-200 (2003).
Stephan, C.N. and R. Arthur: Assessing facial approximation accuracy: how do resemblance ratings of disparate faces compare to recognition tests?. Forensic Sci. Int., 159, 159-63 (2006).

Stephan, C.N. and J. Cicolini: Measuring the accuracy of facial approximations: A comparative study of resemblance rating and face array methods. J. Forensic Sci., 53, 58-64 (2008).

Stephan, C.N. and J. Cicolini: The reproducibility of facial approximation accuracy results generated from photo-spread tests. Forensic Sci. Int., 201, 133-137 (2010).

Tedeschi-Oliveira, S.V., T.L. Beaini and R.F. Melani: Forensic facial reconstruction: Nasal projection in Brazilian adults. Forensic Sci. Int., 266, 123-129 (2016).

Turner, W.D., R.E.B. Brown, T.P. Kelliher, P.H. Tu, M.A. Taister and K.W.P. Miller: A novel method of automated skull registration for forensic facial approximation. Forensic Sci. Int., 154, 149-158 (2005).

Utsuno, H., T. Kageyama, K. Uchida, K. Kibayashi, K. Sakurada and K. Uemura: Pilot study to establish a nasal tip prediction method from unknown human skeletal remains for facial reconstruction and skull photo superimposition as applied to a Japanese male populations. J. Forensic Leg. Med., 38, 75-80 (2016).

Wang, J., X. Zhao, C.Mi and I. Raza: The study on facial soft tissue thickness using Han population in Xinjiang. Forensic Sci. Int., 266, 585.e1-e5 (2016).

Wilkinson, C.M.: Computerized forensic facial reconstruction: A review of current systems. Forensic Sci. Med. Pathol., 1, 173-177 (2005).

Wilkinson, C.M.: Facial reconstruction-anatomical art or artistic anatomy?. J. Anat., 216, 235-250 (2010).

Wilkinson, C.M. and S.A. Mautner: Measurement of eyeball protrusion and its application in facial reconstruction. J. Forensic Sci., 48, 1216 (2003).

Wilkinson, C.M., M. Motwani and E. Chiang: The relationship between the soft tissues and the skeletal detail of the mouth. J. Forensic Sci., 48, 728-732 (2003).

Wilkinson, C.M., R.A.H. Neave and D.S. Smith: How important to facial reconstruction are the correct ethnic group tissue depths? Proceedings of the $10^{\text {th }}$ conference of the International Association of Craniofacial Identification. Bari, Italy, pp. 111-121 (2002a).

Wilkinson, C.M., C. Rynn, H. Peters, M.Taister, C.H. Kau and S. Richmond: A blind accuracy assessment of computer-modeled forensic facial reconstruction using computed tomography data from live subjects. Forensic Sci. Med. Pathol., 2, 179-188 (2006).

Wilkinson, C.M.: Virtual sculpture as a method of computerized facial reconstruction. Proceedings of $1^{\text {st }}$. International Conference on Reconstruction of Soft Facial Parts; Potsdam, Germany, pp. 59-63 (2003).

Wilkinson, C.M. and D.K. Whittaker: Juvenile forensic facial reconstruction: A detailed accuracy study. Proceedings of $10^{\text {th }}$ Conference of the International Association of Craniofacial Identification. Bari, Italy, pp. 11-14 (2002b). 